\title{
Modelling the interactive behaviour of users with a medication safety dashboard in a primary care setting
}

DOI:

10.1016/j.jimedinf.2019.07.014

\section{Document Version}

Accepted author manuscript

Link to publication record in Manchester Research Explorer

\section{Citation for published version (APA):}

Yera, A., Muguerza, J., Arbelaitz, O., Perona, I., Keers, R., Ashcroft, D., Williams, R., Peek, N., Jay, C., \& Vigo, M. (2019). Modelling the interactive behaviour of users with a medication safety dashboard in a primary care setting. International journal of medical informatics, 129, 395. https://doi.org/10.1016/j.ijmedinf.2019.07.014

\section{Published in:}

International journal of medical informatics

\section{Citing this paper}

Please note that where the full-text provided on Manchester Research Explorer is the Author Accepted Manuscript or Proof version this may differ from the final Published version. If citing, it is advised that you check and use the publisher's definitive version.

\section{General rights}

Copyright and moral rights for the publications made accessible in the Research Explorer are retained by the authors and/or other copyright owners and it is a condition of accessing publications that users recognise and abide by the legal requirements associated with these rights.

\section{Takedown policy}

If you believe that this document breaches copyright please refer to the University of Manchester's Takedown Procedures [http://man.ac.uk/04Y6Bo] or contact uml.scholarlycommunications@manchester.ac.uk providing relevant details, so we can investigate your claim.

\section{OPEN ACCESS}




\section{Modelling the interactive behaviour of users with a medication safety dashboard in a primary care setting}

Authors: Ainhoa Yera ${ }^{1}$, Javier Muguerza ${ }^{1}$, Olatz Arbelaitz ${ }^{1}$, Iñigo Perona ${ }^{1}$, Richard N Keers ${ }^{2,4}$, Darren M Ashcroft ${ }^{2,4}$, Richard Williams ${ }^{3,4}$, Niels Peek ${ }^{3,4}$, Caroline Jay ${ }^{5}$, Markel Vigo ${ }^{5 *}$

\section{Affiliations:}

1: Faculty of Informatics, University of the Basque Country UPV/EHU, Donostia/San Sebastián, Spain

2: Division of Pharmacy and Optometry, University of Manchester, Manchester, United Kingdom

3: Division of Informatics, Imaging and Data Sciences, University of Manchester, Manchester Academic Health Science Centre, Manchester, United Kingdom

4: NIHR Greater Manchester Patient Safety Translational Research Centre, University of Manchester, Manchester Academic Health Science Centre, Manchester, United Kingdom

5: School of Computer Science, University of Manchester, Manchester, United Kingdom

* Details of the corresponding author:

Markel Vigo, PhD

2.32 Kilburn Building, Oxford Road

School of Computer Science, University of Manchester

M13 9PL, Manchester, UK

Telephone: $+44(0) 1612750143$

Email: markel.vigo@manchester.ac.uk

Keywords: Patient Safety, Primary Health Care, Supervised Machine Learning, User modelling, Human-Computer Interaction

Word count: 4,766 


\section{ABSTRACT}

Objective: To characterise the use of an electronic medication safety dashboard by exploring and contrasting interactions from primary users (i.e. pharmacists) who were leading the intervention and secondary users (i.e. non-pharmacist staff) who used the dashboard to engage in safe prescribing practices.

Materials and methods: We conducted a 10-month observational study in which 35 health professionals used an instrumented medication safety dashboard for audit and feedback purposes in clinical practice as part of a wider intervention study. We modelled user interaction by computing features representing exploration and $d w e / l$ time through user interface events that were logged on a remote database. We applied supervised learning algorithms to classify primary against secondary users.

Results: We observed values for accuracy above 0.8 , indicating that $80 \%$ of the time we were able to distinguish a primary user from a secondary user. In particular, the Multilayer Perceptron (MLP) yielded the highest values of precision (0.88), recall $(0.86)$ and F-measure (0.86). The behaviour of primary users was distinctive in that they spent less time between mouse clicks (lower dwell time) on the screens showing the overview of the practice and trends. Secondary users exhibited a higher dwell time and more visual search activity (higher exploration) on the screens displaying patients at risk and visualisations.

Discussion and conclusion: We were able to distinguish the interactive behaviour of primary and secondary users of a medication safety dashboard in primary care using timestamped mouse events. Primary users were more competent on population health monitoring activities, while secondary users struggled on activities involving a detailed breakdown of the safety of patients. Informed by these findings, we propose workflows that group these activities and adaptive nudges to increase user engagement. 


\section{INTRODUCTION}

Clinicians are increasingly engaging in quality improvement activities to enhance patient safety in health care settings. Often, these proactive and anticipatory approaches to care management have been facilitated by the availability of data from electronic health records and enable identification of individuals in local communities whose health may be at risk. Medical dashboards display population data and are being used to monitor the health of communities and support clinicians in decisionmaking activities [1]. A few examples highlight the benefits of the visual nature of dashboards, including successful interventions for diabetes care [2] and management of alerts triggered by drug-drug interactions [3]. Typically, medical dashboards display data in a tabular fashion and contain images, charts, numeric and textual information that often tax the perception and cognition of their users [4]. While dashboards may help to alleviate information overload, paradoxically, they may also contribute to this problem by cluttering the screen with information and widgets - some have coined this phenomenon as the "blizzard of dashboards" [5].

Information overload and substandard usability are well-known problems for electronic health record (EHR) systems $[6,7]$. To address this problem, usability guidelines that are sensitive to specific clinical settings and their typical tasks have been derived from general-purpose guidelines $[8,9]$. Similar usability guidelines have also been formulated for medical dashboards [10]. While implementing usability guidelines may address some of the most prominent and critical usability issues, users still feel overwhelmed by the amount of information on the screen. As a result, the interaction of clinicians with health data is not as smooth as expected, and existing evidence indicates that, in order to accomplish their tasks effectively, they demand 'just the right amount' of information [4].

Consequently, it has been suggested that personalised and adaptive user interface capabilities should be implemented to: mitigate the complexity of audit and feedback 
interventions [11]; address information overload in electronic medical records [12]; and improve the effectiveness of clinical tools for decision making [13]. When it comes to medical dashboards, this is not without difficulties in that adapting the user interface to the user's needs calls not only for eliciting such needs, but also taking their skills and expertise into consideration [14]. Yet, detecting the skills and expertise of users (i.e. competence) that will inform the adaptations is particularly challenging due to the evolving nature of knowledge acquisition. This suggests that systems that adapt to the skills and expertise of the users should track competence automatically and unobtrusively.

In this paper, we address this problem by computing proxies of competence on two cohorts of users of a medication safety intervention: a group of 10 pharmacists who led the intervention (i.e. primary users) and a group of 25 non-pharmacist staff (i.e. secondary users). We provide evidence that it is possible to classify different users' interaction with a medication safety dashboard using features including mouse clicks, mouse hovers and timestamps.

\section{The Salford Medication Safety Dashboard (SMASH) Intervention}

The SMASH intervention aims to determine whether the implementation of a pharmacist-led complex intervention reduces the incidence of potentially hazardous prescribing and medication monitoring practices in primary care across Salford, UK [15]. The SMASH dashboard was implemented in 2016, and the quantitative evaluation of the impact on rates of potentially hazardous prescribing is ongoing with results expected in 2019. A concurrent qualitative process evaluation of the SMASH intervention has also been published [16].

The SMASH intervention is comprised of two main components: a web-based interactive dashboard that highlights patients exposed to potentially hazardous prescribing in general practices, and dedicated clinical pharmacist support involving collaborative working with practice staff to resolve hazardous prescribing cases and 
prevent their future occurrence using root cause analysis. The SMASH intervention follows that of the landmark pharmacist-led information-technology based intervention (PINCER) trial [17], but the incorporation of the interactive dashboard is novel.

The SMASH dashboard was co-designed with key stakeholders [18] and incorporates a refined set of 13 prescribing safety indicators which have previously been applied to measure the rate of potentially hazardous prescribing and medication monitoring [19, 20]. For instance, the dashboard identifies all patients' with a history of peptic ulcer who have been prescribed a non-steroidal antiinflammatory drug (NSAID; e.g. ibuprofen) without co-prescription of gastroprotective medication, which places them at risk of gastro-intestinal bleeding, a major adverse event with high mortality rates. The dashboard displays summary statistics for each of the indicators, counting how many patients are currently at risk in a given practice and relating those numbers to previous episodes and other practices. Also, pharmacists and general practitioners (GPs) can view which patients are currently at risk for each indicator. The dashboard is deployed in Salford, a city in the Greater Manchester conurbation, comprising a population of 270,000 served by primary care with additional linkage to secondary care records.

As shown in Figure 1 the user interface of SMASH is divided into seven screens or views: (S1) a landing page containing a tabular overview of a given practice including the size of the practice and the number of patients affected by more than one indicator; (S2) a table view displaying the number of patients who are affected by the indicators, their severity, the number of eligible patients and the percentage of patients who are affected. Indicators can be contraindications between drugs and conditions (e.g. chronic kidney disease and NSAIDs) or between drugs, habits and demographics; (S3) graph-based visualisations displaying the incidence of indicators as time-series. Clicking on the number of patients at risk on the table view (S2) leads 
to (S4) a list of patients at risk for a specific indicator, while clicking on the link 'Patients affected by more than one indicator' on the overview page (S1) leads to S5, which is a patient list similar to S4 but containing only those patients that are affected by more than one indicator. S6 displays the trends for a given indicator over time and S7 contains information and pointers to the medical literature about why a certain indicator is considered a risk.

\section{Aims and Objectives}

Pharmacists, who were the primary users of the electronic dashboard and led on the delivery of the SMASH intervention, worked with secondary dashboard users (GPS and other practice staff) to acknowledge the value of the intervention and integrate it into clinical practice. For primary users, the dashboard was an essential tool to fulfil their key responsibility: i.e. safe prescribing of medication. Secondary users were given access to the dashboard to facilitate multidisciplinary engagement around safe prescribing and continue working with the dashboard to sustain safe prescribing practices.

In the qualitative evaluation of the intervention, primary users indicated that the dashboard added value to their work, while secondary users reported some resistance to engage with it, as some perceived the dashboard was owned by primary users. These attitudes had implications for engagement in that primary users engaged more with the intervention than their colleagues [16]. This is important because the literature concerning engagement at the workplace suggests that those individuals who are engaged are more competent and perform better [21, 22]. This finding has been confirmed in a variety of settings, including healthcare [23].

Therefore, the main goal of this study was to explore whether we could model the interactive behaviour in terms of competence of two groups of electronic dashboard users who reported different levels of engagement in the qualitative evaluation of the SMASH intervention. If the answer to this question were positive, this would allow us 
to examine how the SMASH dashboard is being used and identify distinctive interactive behaviours, and how this may then inform our understanding of the use of medical dashboards in general [1].

Figure 1. Screenshots of the SMASH dashboard (from the top-left to bottom right): practice overview (S1), tabular view of the safety indicators (S2), the visualisation of the safety indicators (S3), the list of patients at risk (S4/S5), indicator trends (S6) and screen containing evidence about why an indicator is a safety hazard (S7)

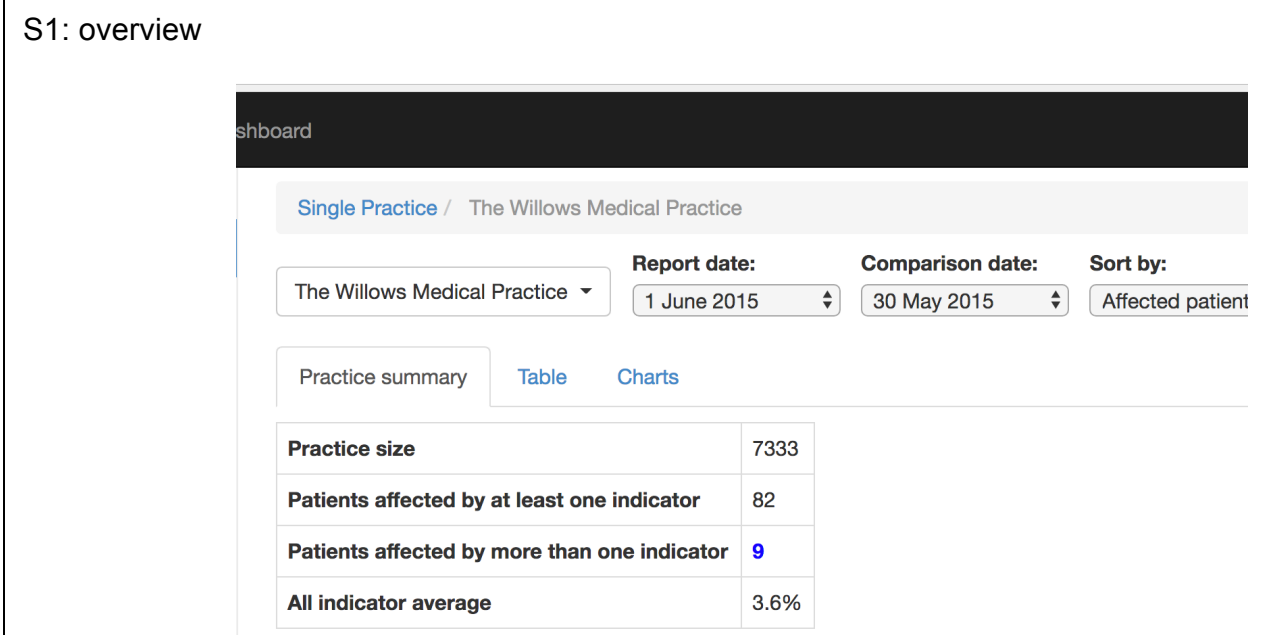

S2: table view

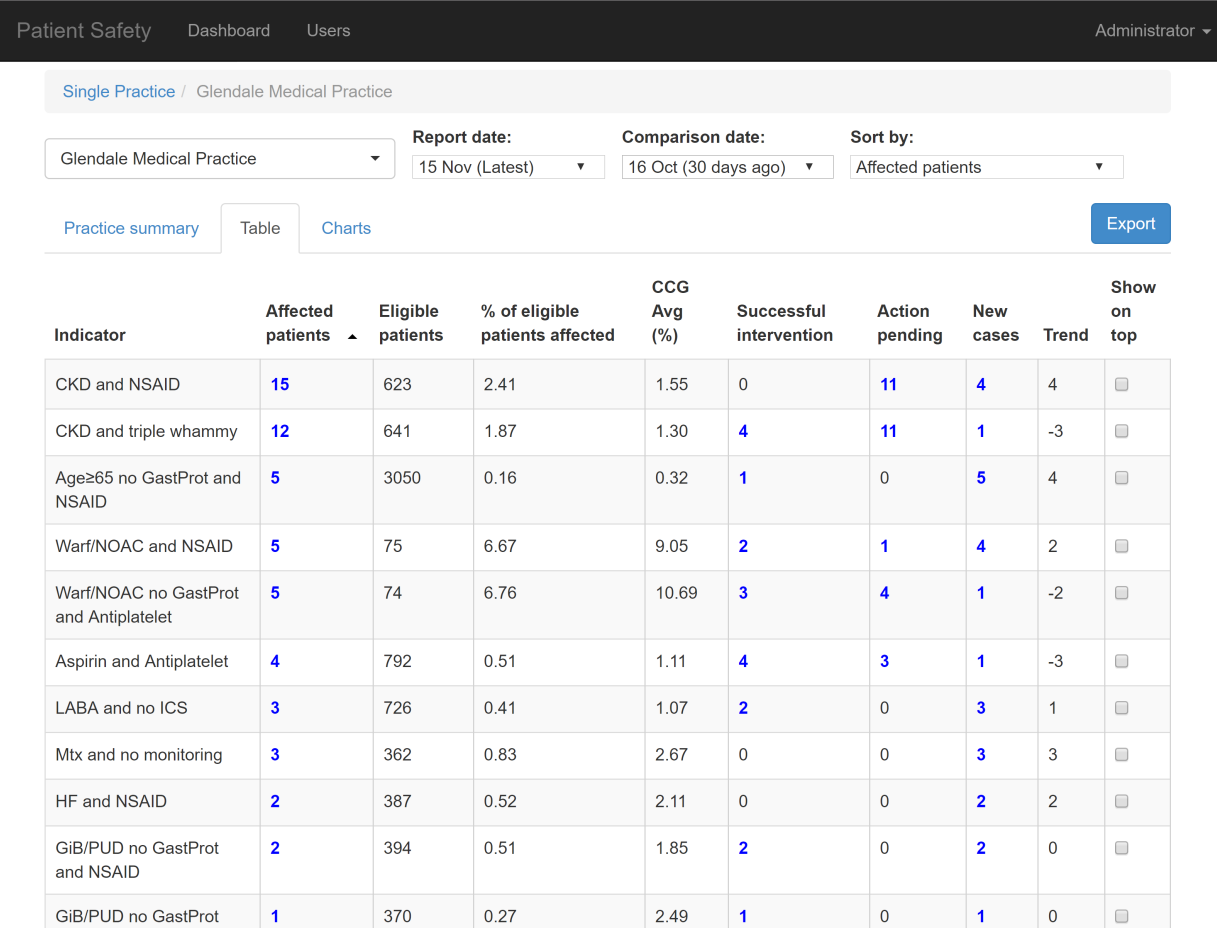




\section{S6: trends}

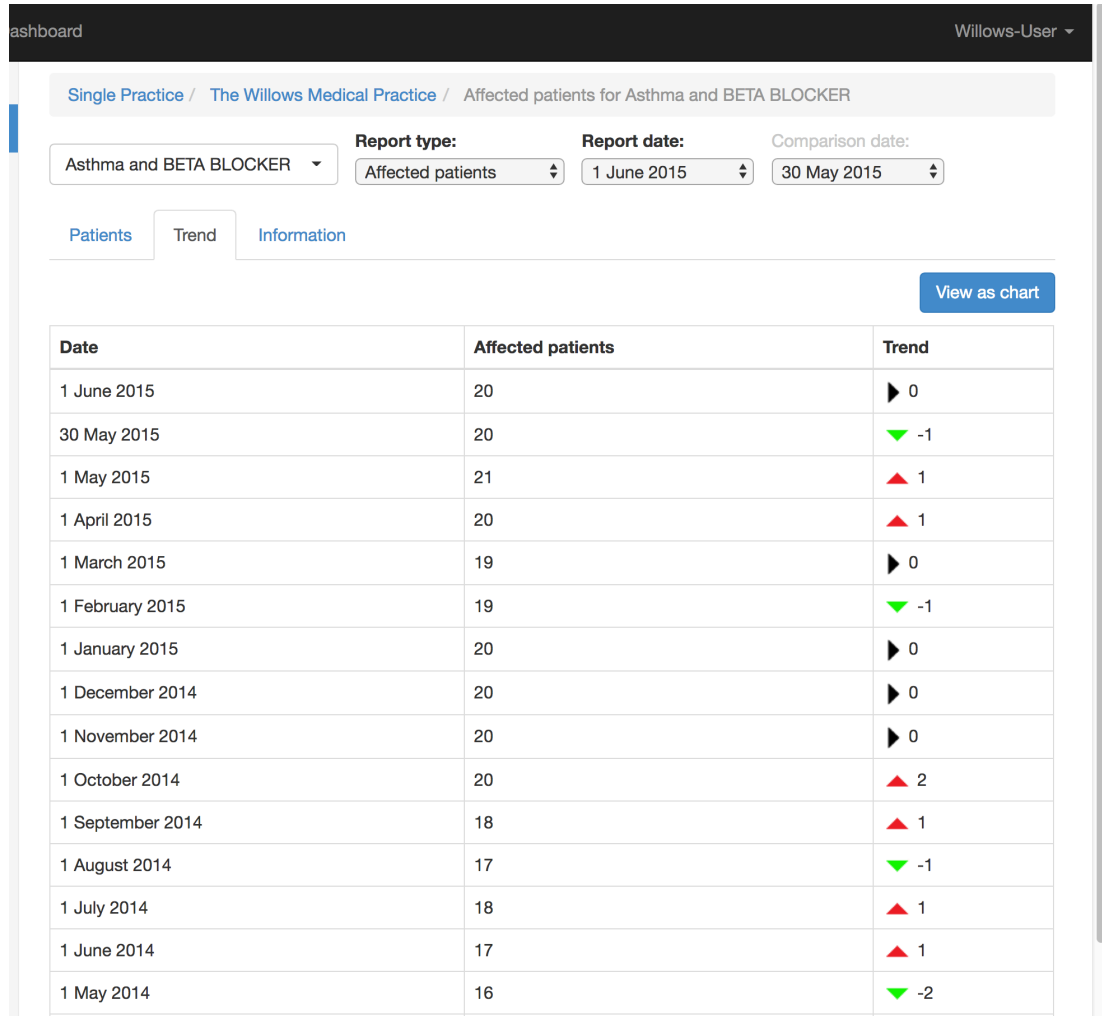

S7: indicator information

Patient Safety Dashboard Users

Single Practice / Glendale Medical Practice / Affected patients for Asthma and BETA BLOCKER

Asthma and BETA BLOCKER V Report type: $\begin{array}{llll} & \text { Report date: } & \text { Comparison date: } \\ \text { Affected patients } & \end{array}$
Patients Trend Information

Patients with a history of asthma who have been prescribed a $\beta$ blocker What is the risk to patients?

In susceptible patients $\beta$ blockers can precipitate acute attacks of bronchospasm or worsen daily symptoms resulting in mortality or low grade morbidity respectively. The BNF advises that " $\beta$ blockers should be avoided in patients with a history of asthma or bronchospasm; if there is no alternative, a cardioselective $\beta$ blockers can be used with extreme caution under specialist supervision. Atenolol, bisoprolol, metoprolol, nebivolol, and (to a lesser extent) acebutolol, have less effect on the $\beta_{2}$ (bronchial) receptors and are, therefore, relatively cardioselective, but they are not cardiospecific. They have a lesser effect on airways resistance but are not free of this side effect". The Committee on Safety of Medicines ${ }^{1}$ issued the following advice: "... $\beta$ blockers, even those with apparent cardioselectivity, should not be used in patients with asthma or a history of obstructive airways disease, unless no alternative treatment is available. In such cases the risk of inducing bronchospasm should be appreciated and appropriate precautions taken."

What evidence is there that this pattern of prescribing is harmful?

$\beta$ blockers vary in their affinity for $\beta_{1}$ - and $\beta_{2}$-adrenoceptors, and are divided into two groups, cardioselective (affinity for $\beta_{1}$ ), and noncardioselective (affinity for $\beta_{2}$ ). The majority show little selectivity for one receptor over the other, except for bisoprolol (14-fold greater affinity for $\beta_{1}$-adrenoceptors) and timolol, sotalol and propranolol (26-fold, 12 -fold, and 8-fold greater affinity for $\beta_{2}$-adrenoceptors, respectively).

Table 1: Cardioselective and non-cardioselective betablockers

Cardioselective beta-blockers (relative selectivity for $\beta_{1}$ adrenoceptors) ${ }^{2}$

Non Cardioselective beta-blockers (relative selectivity for $\beta_{2}$

Acebutolol (2.4) adrenoceptors) ${ }^{2}$

A........,

Labetalol (2.5

n....... 


\section{METHODS}

\section{Data Collection: Apparatus and Study}

The SMASH dashboard is currently being used in 43 GP practices belonging to the Salford Clinical Commissioning Group. SMASH logs the user interface events triggered by users in a database on the host server. Because SMASH is a mousedriven application, the collected events are mostly mouse clicks and mouse hovers (i.e. the events triggered by an element on a website when the mouse hovers over it). For each user interaction event, the dashboard collects the user name, the identifier of the session (i.e. every time a user logs in, a new session is established), the timestamp, the URL where the event took place, and the specific element on the user interface where the event occurred. We collected interaction data accounting for 57,429 user interface events triggered by 35 users from 11 practices during 10 months between January and October 2016.

\section{Users}

We distinguished two cohorts of users: a group of 10 pharmacists leading the intervention (i.e. primary users) who received 2-3 hours of face-to-face formal structured training based on the training principles of the PINCER trial [24] including an interactive seminar covering the background and rationale of the SMASH project, a guided tour of the SMASH dashboard, and the principles of root cause analysis to identify the cause of problems. The group of secondary users was made of 25 nonpharmacist staff including 8 members of the Clinical Commissioning Group (CCG), 8 GPs, 5 managers, and 4 other including nurses and pharmacy technicians who were trained following similar procedures by the primary users.

\section{Features}

Since the SMASH dashboard is a mouse-driven user interface we computed features including mouse clicks and mouse hovers. 
- Exploration (e). Gaze is known to precede action and existing models are accurate in predicting where users are looking using mouse location [25, 26]. Therefore mouse hovers will be indicators of visual search activities on the screen. We computed the median number of mouse hovers between two consecutive mouse clicks, where higher values suggested more visual search activities. Since higher visual activity is known to be an indicator of less efficient search [27], we expect lower exploration values from those users who were more engaged (i.e. primary users).

- Dwell time $(d)$. Time spent on a screen is an indicator of how effective users are processing information and solving problems. A study found that, on information seeking tasks, longer times were correlated with a lower cognitive ability [28]. Hence we computed the median of the elapsed time between two consecutive mouse clicks, where a click triggers an update of the current view by filtering information or leads to another screen of the dashboard. Since lower dwell time conveys higher efficiency accomplishing tasks, we expect primary users will exhibit lower dwell time.

Using these features, we created two interaction representations. A global interaction representation describing user interaction on all the screens available, which is computed as a vector of two attributes: exploration and dwell time on SMASH, global $=\{(e, d)\}$. The second representation, screens, takes into consideration exploration and dwell time in each of the seven screens of SMASH and is represented as a vector of 14 attributes, screens $=\left\{\left(e_{i}, d_{i}\right) \mid i \in \mathbb{N}, i \leq 7\right\}$.

\section{Analysis}

The interaction data generated by users was retrieved from the database and cleansed, which involved identifying users accessing the platform with different credentials, removing variables that were not necessary for this study and eliminating entries corresponding to software engineers and people testing the platform. 
We analysed the data from two perspectives: users and sessions. In the users approach all the events of each user are compiled in one record irrespective of the sessions (accounting for 35 records, split in two cohorts of primary and secondary users). In the sessions approach, one record contains all the events corresponding to a single session (accounting for 564 records). Since users have to be logged into the SMASH dashboard in order to access the platform, we could have computed session durations by using the total period during which users are logged in. However, this method overestimates the length of the session as long periods of inactivity would be included. Hence, we took a more granular approach by which 20 minutes of inactivity would indicate that the session was finished and another session would start as soon as the activity resumed. This approach is in line with the literature on identifying user sessions [29]. By applying this method, we record a total of 564 sessions: 419 corresponding to primary users $(74 \%)$ and 145 to secondary users $(26 \%)$ distributed as 64 exhibited by CCG staff, 27 by GPs, 32 by general practice managers and 22 by other. The unequal number of sessions confirms what qualitative studies [16] reported on the higher engagement of primary users: $29 \%$ of primary users generated $74 \%$ of the sessions.

Both interaction representations (global and screens) were computed for each user and each session. In both types of analysis, the records are labelled according to the user group of the user (i.e. primary and secondary). Table 1 shows the distribution of users and sessions per user group.

Table 1. Number of users and number of sessions per group

\begin{tabular}{|l|l|l|}
\hline User group & $\mathbf{N}(\%)$ & Sessions (\%) \\
\hline Primary users & $10(29)$ & $419(74)$ \\
\hline Secondary users & $25(71)$ & $145(26)$ \\
\hline GPs & $8(23)$ & $27(5)$ \\
\hline CCG staff & $8(23)$ & $64(11)$ \\
\hline Managers & $5(14)$ & $32(6)$ \\
\hline Other & $4(11)$ & $22(4)$ \\
\hline
\end{tabular}

Following this, we used the Weka machine learning software [30] to classify the different types of users - primary and secondary - using machine learning 
algorithms, and used 10-fold cross-validation (CV) to evaluate the performance of the algorithms. In the sessions approach, since different session observations for the same user are not independent, we also performed an approximately stratified 3-fold $\mathrm{CV}$, splitting the dataset in three folds of similar size and similar primary/secondary proportion but keeping all the sessions of each user in the same fold.

We calculated precision (ratio of users that were correctly classified, out of those who were predicted to belong to a particular class: i.e. primary or secondary), recall (ratio of users that were correctly classified, out of those who belong to a particular class) and f-measure (the harmonic mean of precision and recall).

\section{RESULTS}

\section{The User Perspective}

Table 2 shows values of exploration and dwell time on the global and screens representation where, in general, secondary users exhibit higher values for both features. Then we experimented with different supervised learning algorithms [31] that were selected due to their applicability, relevance and popularity [32]: IBK, (Weka's implementation of the k-Nearest Neighbour algorithm), SMO (Weka's implementation of Support Vector Machines), MLP (Multilayer Perceptron), Naïve Bayes and $\mathrm{J} 48$ (Weka's implementation of the C4.5 algorithm), and two multiple classifier systems including bagging and boosting.

Table 2. Exploration (mouse hovers between clicks) and dwell time (seconds) average from the user perspective, where S1: overview, S2: table view, S3: visualisations, S4: patients at risk, S5: patients affected by more than one indicator, S6: trends, S7: indicator information.

\begin{tabular}{|l|l|l|l|l|l|l|l|l|l|}
\hline Feature & User group & Global & S1 & S2 & S3 & S4 & S5 & S6 & S7 \\
\hline \multirow{2}{*}{$\begin{array}{l}\text { Exploration } \\
\text { (mouse hovers } \\
\text { between clicks) }\end{array}$} & all & 3.4 & 3.84 & 3.21 & 3.29 & 2.83 & 2.34 & 1.87 & 1.6 \\
\cline { 2 - 10 } & primary & 2.9 & 3.7 & 3.3 & 2.75 & 2.5 & 3.5 & 2.7 & 3.4 \\
\hline \multirow{2}{*}{$\begin{array}{l}\text { Dwell time } \\
\text { (seconds) }\end{array}$} & secondary & 3.6 & 3.9 & 3.18 & 3.5 & 2.96 & 1.88 & 1.54 & 0.9 \\
\cline { 2 - 10 } & primary & 3.47 & 8.93 & 2.75 & 16.02 & 3.68 & 6.28 & 2.15 & 7.93 \\
\cline { 2 - 10 } & secondary & 2.23 & 16.88 & 2.82 & 2.54 & 2.10 & 5.88 & 1.59 & 3.27 \\
\hline
\end{tabular}


The dataset was found to be unbalanced as 10 primary users accounted for $29 \%$ of users out of 35 individuals. Table 3 shows the performance of the algorithms where IBK, J48 and Naïve Bayes produce scores for precision, recall and f-measure above 0.80 for the screens representation. Scores were lower when not taking into consideration the particular screens (i.e. global) although the MLP algorithm achieved values above 0.80 for both representations.

Table 3. Precision, recall and f-measure on users per algorithm.

\begin{tabular}{|l|l|l|l|l|}
\hline Algorithm & Representation & Precision & Recall & F-measure \\
\hline \multirow{2}{*}{ AdaBoost } & global & 0.73 & 0.71 & 0.72 \\
\cline { 2 - 5 } & screens & 0.77 & 0.77 & 0.77 \\
\hline \multirow{3}{*}{ IBagging } & global & 0.67 & 0.71 & 0.67 \\
\cline { 2 - 5 } & screens & 0.74 & 0.74 & 0.74 \\
\hline \multirow{3}{*}{ J48 } & global & 0.71 & 0.71 & 0.71 \\
\cline { 2 - 5 } & screens & 0.84 & 0.83 & 0.83 \\
\hline \multirow{2}{*}{ MLP } & global & 0.60 & 0.60 & 0.60 \\
\cline { 2 - 5 } & screens & 0.84 & 0.83 & 0.83 \\
\hline \multirow{2}{*}{ Naïve Bayes } & global & 0.83 & 0.83 & 0.83 \\
\cline { 2 - 5 } & screens & 0.86 & 0.86 & 0.86 \\
\cline { 2 - 5 } & global & 0.77 & 0.74 & 0.75 \\
\hline \multirow{2}{*}{ SMO } & global & 0.90 & 0.89 & 0.89 \\
\cline { 2 - 5 } & screens & 0.51 & 0.71 & 0.60 \\
\hline
\end{tabular}

The scores in Table 3 were useful to the extent that false positives (computed for precision) and false negatives (computed for recall) can be tolerated, which is ultimately dependent on the purpose of the classifiers. While discriminating users (primary vs secondary) is certainly necessary for user modelling purposes, the extraction of the characteristics of each user group provided valuable information that could inform future adaptations. Among the selected classifiers, J48 provides an intuitive interpretation in that the path between the root node and the leaf nodes includes the relational rules whereby different instances get classified [31]. We analysed the structure of the J48 classifier generated with the complete sample for the global and screens representation, using all the available information, because this would be the tree that would be deployed in a real system. 
Figure 2. Graphical representation of a J48 pruned tree for global resulting in 0.6 for precision, 0.6 for recall and 0.6 for $\mathrm{f}$-measure. The circles on the top-right part of each square indicate the number of individuals who fell in this condition while the numbers in the square convey the distribution of these individuals by group.

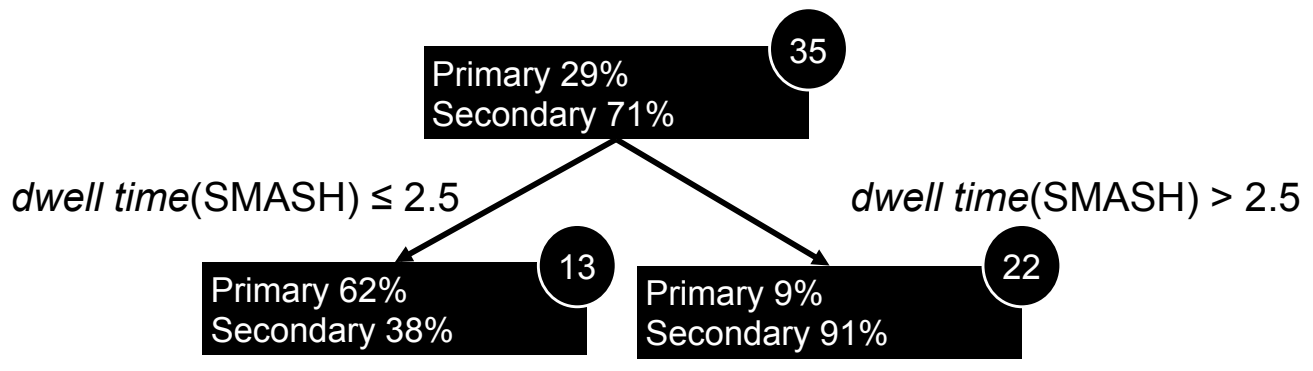

Despite J48 having lower scores than other algorithms for the global representation, dwell time was able to discriminate primary from secondary users in the SMASH dashboard platform. According to the structure of J48's classification in Figure 2, if dwell time was smaller or equal to 2.5 seconds (i.e. the user spends 2.5 seconds or less between mouse clicks), primary users accounted for $62 \%(8 / 13)$ of the users (note that initially primary users comprised $29 \%$ of users), whereas secondary users constituted $91 \%(19 / 22)$ of the individuals (up from the original $71 \%$ ) when dwell time was restricted to observations greater than 2.5 seconds. Note that the average dwell time score when using the SMASH dashboard was $3.47(\mathrm{SD}=1.87)$, which revealed that primary users spent less time between clicks (mean 2.23 seconds) compared to their counterparts (mean 3.97 seconds).

The structure of a J48 pruned tree is illustrated in Figure 3 for the screens representation. The average value for the exploration metric on the indicators information screen $(S 7)$ was 1.6 mouse hovers $(S D=2.31)$. However, primary users' exploration was higher than the average (3.35 mouse hovers) and secondary users exploration ( 0.9 mouse hovers). If exploration was restricted to users with values of 0 - meaning there was no activity on the indicators information screen - secondary users constituted $95 \%(20 / 21)$ from an initial $71 \%$ of the users in this node of the 
classification tree. The remaining 15 users (53\% primary and $47 \%$ secondary) who exhibited some activity in the indicators information screen fell into three conditions.

Figure 3. Graphical representation of a J48 pruned tree for screens resulting in 0.84 for precision, 0.83 for recall and 0.83 for $\mathrm{f}$-measure.

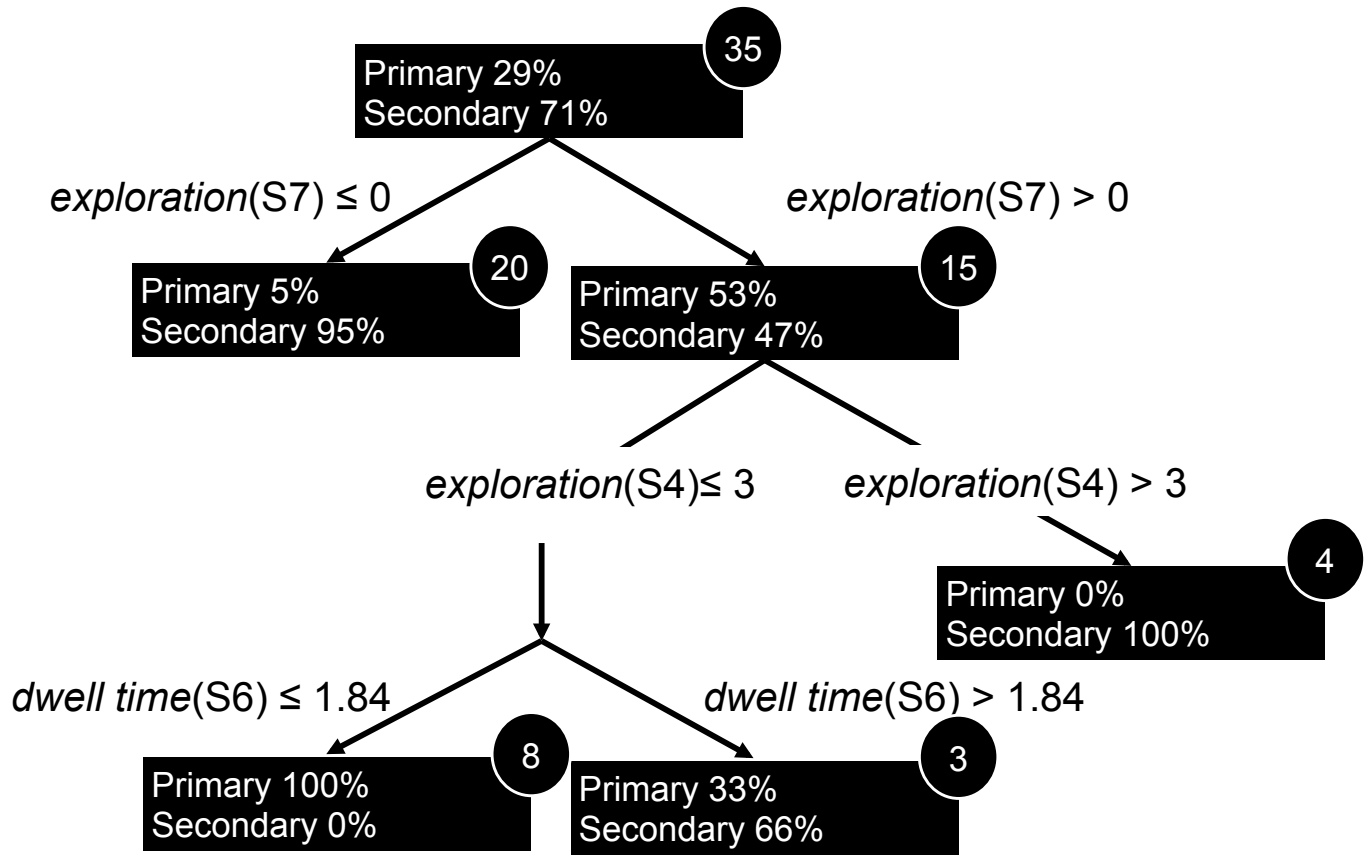

The users whose exploration activity was more than 3 mouse hovers on the screen listing patients affected by potentially hazardous prescribing indicators (S4) were exclusively secondary (4 individuals, accounting for $100 \%$ of users in this node). Note that the average is 2.83 mouse hovers, 2.50 for primary users vs 2.96 for secondary users. If the activity was equal to or less than 3 mouse hovers and, if time spent on the trends screen (S6) was less than or equal to 1.84 seconds, all the users in this node were primary users (accounting for 8 individuals), while when dwell time was greater than 1.84 seconds, one was a primary user and the remaining two were not - note that the average dwell time is 2.15 seconds, 1.59 seconds for primary users $(S D=0.92)$ and 2.38 seconds $(S D=4.91)$ for secondary users. 


\section{The Session Perspective}

Table 4 displays values of exploration and dwell time where, again, primary users exhibit lower values in general, although this behaviour is not consistent across all the screens.

Table 4. Exploration (mouse hovers between clicks) and dwell time (seconds) average on the global and screens representations from the session perspective.

\begin{tabular}{|l|l|l|l|l|l|l|l|l|l|}
\hline Feature & User group & Global & S1 & S2 & S3 & S4 & S5 & S6 & S7 \\
\hline \multirow{5}{*}{ Exploration } & all & 3.79 & 4.18 & 3.43 & 1.05 & 1.94 & 0.79 & 0.62 & 0.47 \\
\cline { 2 - 10 } & primary & 3.65 & 4.08 & 3.52 & 0.81 & 1.82 & 0.87 & 0.71 & 0.53 \\
\cline { 2 - 9 } & secondary & 4.19 & 4.45 & 3.14 & 1.74 & 2.29 & 0.58 & 0.36 & 0.22 \\
\hline \multirow{3}{*}{ Dwell time } & all & 3.28 & 6.81 & 10.01 & 4.6 & 3.03 & 3.4 & 1.05 & 3.35 \\
\cline { 2 - 9 } & primary & 2.85 & 5.53 & 6.97 & 3.59 & 3.1 & 3.84 & 1.23 & 3.55 \\
\cline { 2 - 9 } & secondary & 4.51 & 10.48 & 11.01 & 7.48 & 2.84 & 2.14 & 0.53 & 2.75 \\
\hline
\end{tabular}

Table 5 shows the scores obtained when the data was analysed from a session perspective. Since we have recurrent users their sessions are not independent, we performed an approximately stratified 3-fold CV, splitting the dataset in three folds of similar size and similar primary/secondary proportion but keeping all the sessions of each user in the same fold.

The performance of the classifiers is lower in this analysis, and the best performing algorithm is Bagging. As expected, the performance of the 3-fold CV is slightly worse than the 10 -fold CV. Note that this would be a worst but unrealistic case since having exclusively new users in the test set would mean that there would not be recurrent users. Therefore, if the system was to be exploited in a real context, where most users would be expected to regularly interact with the dashboard, we could expect the performance of the proposed classification system to be closer to the 10 -fold cross-validation approach, being the result somewhere in between the two approaches. 
Table 5. Precision, recall and f-measure on sessions per algorithm.

\begin{tabular}{|c|c|c|c|c|c|}
\hline Algorithm & $\mathrm{CV}$ & Analysis & Precision & Recall & F-measure \\
\hline \multirow[t]{4}{*}{ AdaBoost } & \multirow[t]{2}{*}{ 3-fold } & global & 0.65 & 0.73 & 0.68 \\
\hline & & screens & 0.73 & 0.71 & 0.67 \\
\hline & \multirow[t]{2}{*}{ 10-fold } & global & 0.68 & 0.73 & 0.69 \\
\hline & & screens & 0.73 & 0.76 & 0.72 \\
\hline \multirow{4}{*}{ Bagging } & \multirow[t]{2}{*}{ 3-fold } & global & 0.71 & 0.72 & 0.69 \\
\hline & & screens & 0.68 & 0.70 & 0.68 \\
\hline & \multirow[t]{2}{*}{ 10-fold } & global & 0.70 & 0.74 & 0.70 \\
\hline & & screens & 0.77 & 0.78 & 0.77 \\
\hline \multirow[t]{4}{*}{ IBK } & \multirow[t]{2}{*}{ 3-fold } & global & 0.63 & 0.64 & 0.63 \\
\hline & & screens & 0.68 & 0.69 & 0.68 \\
\hline & \multirow{2}{*}{ 10-fold } & global & 0.65 & 0.65 & 0.65 \\
\hline & & screens & 0.72 & 0.73 & 0.73 \\
\hline \multirow[t]{4}{*}{$\mathrm{J} 48$} & \multirow[t]{2}{*}{ 3-fold } & global & 0.59 & 0.74 & 0.63 \\
\hline & & screens & 0.69 & 0.69 & 0.68 \\
\hline & \multirow[t]{2}{*}{ 10-fold } & global & 0.67 & 0.72 & 0.68 \\
\hline & & screens & 0.73 & 0.76 & 0.73 \\
\hline \multirow[t]{4}{*}{ MLP } & \multirow[t]{2}{*}{ 3-fold } & global & 0.70 & 0.73 & 0.69 \\
\hline & & screens & 0.70 & 0.74 & 0.70 \\
\hline & \multirow[t]{2}{*}{ 10-fold } & global & 0.67 & 0.72 & 0.68 \\
\hline & & screens & 0.71 & 0.75 & 0.71 \\
\hline \multirow[t]{4}{*}{ Naive Bayes } & \multirow[t]{2}{*}{ 3-fold } & global & 0.64 & 0.71 & 0.64 \\
\hline & & screens & 0.64 & 0.48 & 0.47 \\
\hline & \multirow[t]{2}{*}{ 10-fold } & global & 0.61 & 0.71 & 0.64 \\
\hline & & screens & 0.69 & 0.59 & 0.62 \\
\hline \multirow[t]{4}{*}{ SMO } & \multirow[t]{2}{*}{ 3-fold } & global & 0.55 & 0.74 & 0.63 \\
\hline & & screens & 0.58 & 0.73 & 0.63 \\
\hline & \multirow[t]{2}{*}{ 10-fold } & global & 0.55 & 0.74 & 0.63 \\
\hline & & screens & 0.55 & 0.74 & 0.63 \\
\hline
\end{tabular}

We analysed the structure of the J48 classifier generated with the complete sample for the screens representation. As mentioned above, the performance of the tree would be the one estimated with the 10 -fold $\mathrm{CV}$ for regular users and 3-fold CV for new users. The rightmost branch of the J48 classification structure in Figure 4 indicates that $81 \%$ of the sessions belonged to secondary users when there were more than 3.5 mouse hovers on the visualisations screen ( 1.74 mouse hovers by secondary users vs 0.81 by primary users on S3), 0 mouse hovers on the screen containing information about prescribing safety indicators $(0.22$ mouse hovers by secondary users vs 0.53 by primary users on S7) and users spent less than 4.47 seconds between clicks on the screen showing patients at risk (S5), where 3.40 seconds were spent on average. 
Figure 4. Graphical representation of the most relevant nodes of the J48 pruned tree for screens resulting in 0.73 for precision, 0.76 for recall and 0.73 for $\mathrm{f}$-measure.

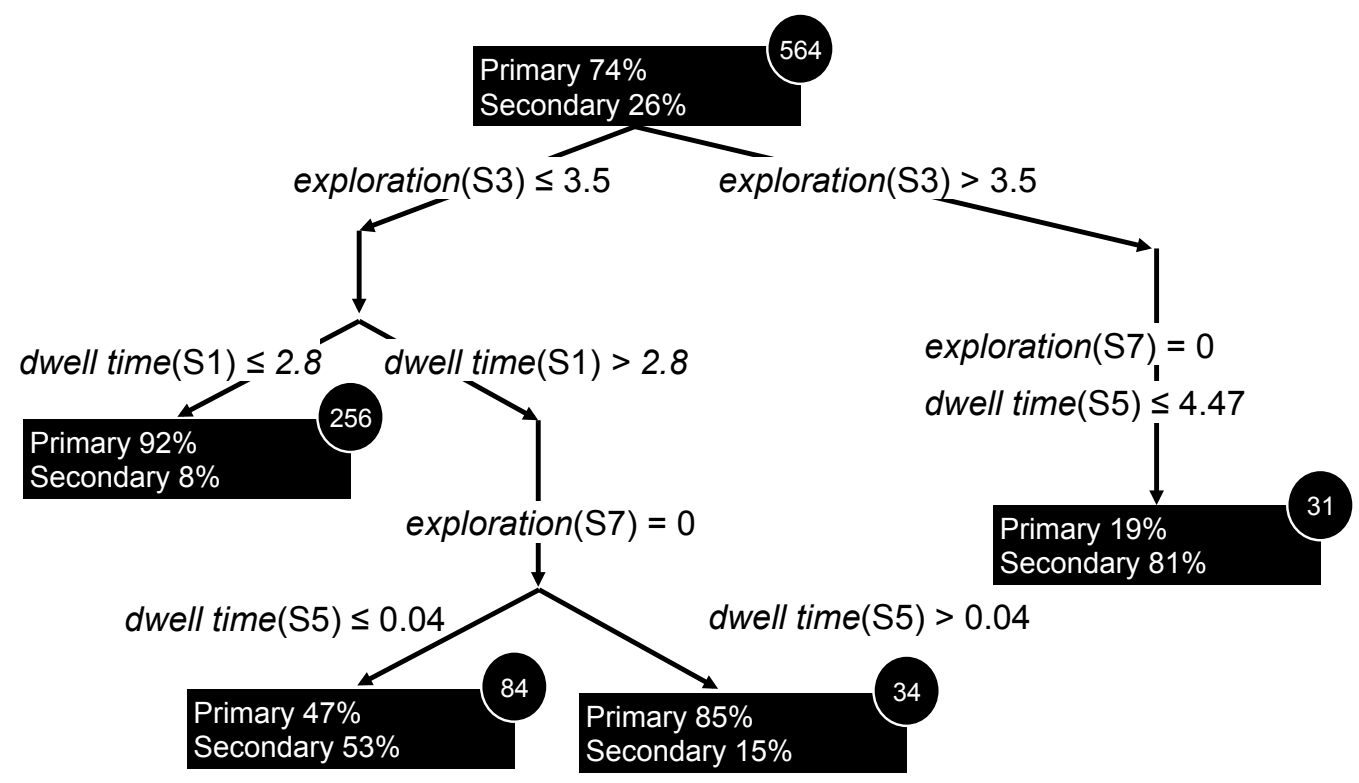

$92 \%$ of the sessions belonged to primary users when the number of mouse hovers between clicks on visualisations (S3) was less than or equal to 3.5 mouse hovers and the time spent on the landing page (S1) was less than or equal to 2.8 seconds (the average time was 6.8 seconds). When users spent more than 2.8 seconds on the landing page, the screen containing information about patients at risk (S5) was decisive to classify the users: when they spent less than or equal to the cut-off value ( 0.04 seconds, average was 3.4 seconds), $53 \%$ of the sessions were exhibited by secondary users, whereas $85 \%$ of the sessions belonged to primary users otherwise.

\section{DISCUSSION}

We found that our initial expectations that primary SMASH dashboard users would exhibit lower values for dwell time and exploration were met in both representations (i.e. global and screens) and perspectives - see users in Table 2 and sessions in Table 4. When considering the classification algorithms, these expectations were also met as exhibited by the values of cut-off points in the classification trees in Figures 2-4. In general, descriptive statistics and classification algorithms confirm 
that lower dwell time and exploration was characteristic of those who engaged more with the intervention (i.e. primary users).

Lower values of dwell time were key to distinguish primary from secondary dashboard users in the global representation (Figure 2), in the screen that displayed the prescribing safety indicator trends of a given practice (S6 in Figure 3) and on the landing page containing the overview of the practice (S1 in Figure 4). The behaviour of secondary users was characterised by higher dwell time on the screen that displays patients at risk (S4 in Figure 3) and higher values of exploration on the visualisations screen (S3 in Figure 4). It should be noted that the usage of the dashboard is not exclusive to the user groups, but what characterises the user groups is how this usage is exhibited. Specifically, these findings suggest that secondary users exhibit characteristic behaviours on screens showing a detailed breakdown of the safety of patients (patients at risk and visualisations), while primary users are characterised by their use of the SMASH dashboard to monitor population health on screens showing the overview of the practice and trends.

There were exceptions: higher dwell time was characteristic of primary users on the screen, showing patients affected by more than one indicator (see S5 in Table 4 and Figure 4). We know from an ongoing study [33] that primary dashboard users spend most of their time on S4/S5 (i.e. patients at risk) because they would check these patients' electronic health records and perhaps make some phone calls. Additionally, lower values of exploration corresponded to secondary users on the screen describing particular indicators (S7 in Table 2 and Table 4, and Figure 3 and Figure 4). It is worth noting that in these instances the cut-off values were close to 0 suggesting that secondary users did not exhibit low dwell time and exploration values because they were more effective, but because they did not access those screens. This implies that, when the functionalities are accessed, dwell time and exploration serve as proxies that characterise the interactive behaviour in the SMASH dashboard 
in that they discriminate primary users with high accuracy. For each algorithm, the screens representation performs better than the global one, which means that including the screens in the modelling has added value.

These findings suggest that the two user groups (primary and secondary users) have different characteristic behaviours when interacting with the SMASH dashboard. These interactive behaviours, which are modelled using features that are proxies of competence, make the two user groups distinguishable. Lower values of dwell time are indicators of users being more effective in processing information and solving problems [28], which suggests that primary users were more competent carrying out overseeing tasks in the SMASH dashboard. Since longer visual activity conveyed by exploration is known to be an indicator of less efficient search [27], we attribute higher exploration values observed in secondary users to lower levels of engagement and, consequently, lower performance. This is in line with the literature that indicates that those who are more engaged perform better [21, 22].

Two design recommendations emerge from these outcomes. Since the perceived lack of competence is a barrier to use this kind of interventions [34], we could monitor the competence of SMASH dashboard users (and similar interventions) and intervene if needed. Individuals belonging to groups of users who are less engaged (and are less competent) could be given support using tailored educational nudges to encourage their learning. They could be provided with personalised messages about their performance with respect to their peers, which could help to challenge their perceptions if they underestimated themselves and increase self-efficacy. These nudges could potentially be delivered by retrieving the current URL and keeping track of mouse events to compute dwell time and exploration, which can be carried out in real time in the browser. Since this method does not require to remote storage of interaction or personal data, user confidentiality and privacy are respected, removing potential barriers for acceptance of such systems by prospective users [35]. The 
second recommendation is about adapting the workflows in the SMASH dashboard according to the characteristic use of the two groups. Informed by the stereotypical uses of the dashboard, SMASH should facilitate workflows for (a) monitoring population health and (b) for a more detailed analysis of individuals at risk, by grouping the screens accordingly. Transitions between these two workflows should also be possible by mapping an analogy of the information visualisation mantra (i.e. overview, filtering, details-on-demand) [36] into dashboards for managing population health through progressive disclosure principles: monitoring population health, filtering, breakdown-of-data on demand.

\section{Methodological Considerations}

Primary users comprised $29 \%$ of the users in the study while their sessions accounted for $74 \%$ of the total sessions. This means that since we had more recurring sessions from primary users we might have confounded learning effects in the user perspective. While our conclusion still holds (primary users are more efficient carrying out overseeing activities in the SMASH dashboard) the reason they are more confident could be a result not only of their engagement with the intervention but also to their expertise due to the fact they were pharmacists. Nevertheless, we found that while having this expertise is beneficial, it is not essential to engaging with the intervention [33].

\section{CONCLUSION}

This study successfully modelled the interactive behaviour of two different cohorts of electronic dashboard users. In addition to the explicit differences derived from the descriptive analysis, we identified the differences that characterised the two groups in terms of their interactive behaviours. These differences are important to understand everyday use of the SMASH dashboard where primary pharmacist users are more competent on screens that provide summary and trends information, while secondary general practice staff users are less competent on screens containing a 
detailed breakdown of the data. We propose workflows that encompass these activities in a coherent sequence and personalised educational nudges to foster engagement.

The contributions of the paper are twofold: a methodological contribution suggests that it is feasible to characterise the interactive behaviour of users in a medication safety dashboard using user interaction events. An empirical contribution advances into our understanding of how medical dashboards are used by health care stakeholders, an area which remains largely unexplored and is key to perform adaptations that cater for the users' ability to perceive, process and make data actionable [1].

\section{FUNDING}

This work was partially funded by the National Institute for Health Research Greater Manchester Patient Safety Translational Research Centre (NIHR Greater Manchester PSTRC) and the NIHR Manchester Biomedical Research Centre. The views expressed are those of the authors and not necessarily those of the NHS, the NIHR or the Department of Health and Social Care. Funded by the University of the Basque Country UPV/EHU (grant PIF15/143); by the research group ADIAN that is supported by the Department of Education, Universities and Research of the Basque Government, (grant IT980-16); and by the Ministry of Economy and Competitiveness of the Spanish Government, co-founded by the ERDF (PhysComp project, TIN201785409-P).

\section{ETHICS}

Ethical approval for the study was granted by the NHS National Research Ethics Service (reference 15/NW/0792).

\section{COMPETING INTERESTS}

None. 


\section{DATA SHARING}

The interaction event logs analysed in this work are available at https://doi.org/10.5281/zenodo.1296337

\section{CONTRIBUTION}

MV, CJ and NP conceived the study. The SMASH electronic medication safety dashboard was developed by RW and RNK, and engineered by RW. MV cleansed the raw log data. AY, JM, OA and IP analysed the data. MV wrote the manuscript. All authors critically edited the manuscript. All authors approved the manuscript.

\section{REFERENCES}

1 Dowding D, Randell R, Gardner $\mathrm{P}$, et al. Dashboards for improving patient care: Review of the literature. Int J Med Inform 2015; 84:87-100.

2 Dagliati A, Sacchi L, Tibollo V, et al. A dashboard-based system for supporting diabetes care, J Am Med Inform Assoc 2018;25:538-47.

3 Simpao AF, Ahumada LM, Desai BR, et al. Optimization of drug-drug interaction alert rules in a pediatric hospital's electronic health record system using a visual analytics dashboard, J Am Med Inform Assoc 2015,22:361-69.

4 Yera A, Muguerza J, Arbelaitz $\mathrm{O}$, et al. Inferring Visual Behaviour from User Interaction Data on a Medical Dashboard. In: Proceedings of the 2018 International Conference on Digital Health 2018:55-59.

5 Kalra D, Buchan I, Paton N. Three Gurus of Big Data. The Translational Scientist 2016;0816:401. https://thetranslationalscientist.com/issues/0816/three-gurus-of-bigdata/ (accessed 7 Jun 2018)

6 Middleton B, Bloomrosen M, Dente MA, et al. Enhancing patient safety and quality of care by improving the usability of electronic health record systems: recommendations from AMIA, J Am Med Inform Assoc 2013;20 e2-e8. 
7 Ratwani RM, Fairbanks RJ, Hettinger AZ, et al. Electronic health record usability: analysis of the user-centered design processes of eleven electronic health record vendors, J Am Med Inform Assoc 2015;22:1179-82.

8 Kushniruk AW, Patel VL. Cognitive and usability engineering methods for the evaluation of clinical information systems. J Biomed Inform 2004;37:56-76.

9 Zhang J, Walji MF. TURF: Toward a unified framework of EHR usability. J Biomed Inform 2011;44:1056-67.

10 Brown B, Balatsoukas P, Williams R, et al. Interface design recommendations for computerised clinical audit and feedback: Hybrid usability evidence from a researchled system. Int J Med Inform 2016;94:191-206.

11 Landis-Lewis Z, Brehaut JC, Hochheiser H, et al. Computer-supported feedback message tailoring: theory-informed adaptation of clinical audit and feedback for learning and behavior change. Implement Sci 2015;10:12.

12 Zahabi M, Kaber DB, Swangnetr M. Usability and Safety in Electronic Medical Records Interface Design: A Review of Recent Literature and Guideline Formulation. Hum Factors 2015;57:805-34.

13 Brehaut JC, Colquhoun HL, Eva KW, et al. Practice feedback interventions: 15 suggestions for optimizing effectiveness. Ann Inter Med 2016;164:435-41.

14 Dowding D, Merrill JA, Onorato N, et al. The impact of home care nurses' numeracy and graph literacy on comprehension of visual display information: implications for dashboard design. J Am Med Inform Assoc 2018;25:175-82.

15 Williams R, Keers R, Gude W, et al. SMASH! The Salford medication safety dashboard. J Innov Health Inform 2018;25:183-93.

16 Jeffries M, Keers RN, Phipps DL, et al. Developing a learning health system: Insights from a qualitative process evaluation of a pharmacist-led electronic audit and 
feedback intervention to improve medication safety in primary care. PLoS One 2018;13:e0205419.

17 Avery AJ, Rodgers S, Cantrill JA, et al. A pharmacist-led information technology intervention for medication errors (PINCER): a multicentre, cluster randomised, controlled trial and cost-effectiveness analysis. Lancet 2012;379:1310-19.

18 Keers RN, Williams R, Davies C, et al. Improving medication safety in primary care: developing a stakeholder-centred electronic prescribing safety indicator dashboard. Pharmacoepidemiology and Drug Safety 2015:Online Supp.

19 Akbarov A, Kontopantelis E, Sperrin M, et al. Primary care medication safety surveillance with integrated primary and secondary care electronic health records: a cross-sectional study. Drug Saf 2015;38:671-82.

20 Stocks SJ, Kontopantelis E, Akbarov A, et al. Examining variations in prescribing safety in UK general practice: cross sectional study using the Clinical Practice Research Datalink. BMJ 2015;351:h5501.

21 Rich BL, Lepine JA, Crawford ER. Job engagement: Antecedents and effects on job performance. Acad Manage J 2010;53:617-35.

22 Christian MS, Garza AS, Slaughter JE. Work engagement: A quantitative review and test of its relations with task and contextual performance. Pers Psychol 2011;64:89-136.

23 Spence Laschinger HK, Wilk $\mathrm{P}$, Cho J, et al. Empowerment, engagement and perceived effectiveness in nursing work environments: does experience matter? J Nurs Manag 2009;17:636-46.

24 Sadler S, Rodgers S, Howard R, et al. Training pharmacists to deliver a complex information technology intervention (PINCER) using the principles of educational outreach and root cause analysis. Int J Pharm Pract 2014;22:47-58. 
25 Guo Q, Agichtein E. Towards predicting web searcher gaze position from mouse movements. In: $\mathrm{CHI}$ '10 Extended Abstracts on Human Factors in Computing Systems 2010:3601-3606.

26 Navalpakkam V, Jentzsch L, Sayres R, et al. Measurement and modeling of eyemouse behavior in the presence of nonlinear page layouts. In: Proceedings of the 22nd international conference on World Wide Web 2013:953-964.

27 Ehmke C, Wilson S. Identifying web usability problems from eye-tracking data. In: Proceedings of the 21st British $\mathrm{HCl}$ Group Annual Conference on People and Computers 2007:119-128.

28 Chin J, Fu WT, Kannampallil T. Adaptive information search: age-dependent interactions between cognitive profiles and strategies. In: Proceedings of the SIGCHI Conference on Human Factors in Computing Systems 2009:1683-1692.

29 Heer J, Chi EH. Separating the swarm: categorization methods for user sessions on the web. In: Proceedings of the SIGCHI Conference on Human Factors in Computing Systems 2002:243-50.

30 Hall M, Frank E, Holmes G, et al. The WEKA data mining software: an update. SIGKDD Explor NewsI 2009:10-18.

31 Witten IH, Frank E, Hall M, et al. Data Mining: Practical machine learning tools and techniques. Morgan Kaufmann 2016.

$32 \mathrm{Wu}$ X, Kumar V, Ross Quinlan J, et al. Top 10 algorithms in data mining. Knowl Inf Syst 2008;14:1-37.

33 Jeffries M, Gude WT, Keers RN, et al. Understanding the utilisation of a novel interactive electronic medication safety dashboard by pharmacists and clinicians in general practice: a mixed methods study (Paper under review) 
34 Jeffries M, Phipps D, Howard R, et al. Understanding the implementation and adoption of an information technology intervention to support medicine optimisation in primary care: qualitative study using strong structuration theory. BMJ Open 2017;7:e014810.

35 Angulo J, Ortlieb M. 2015. "WTH...??" Experiences, Reactions, and Expectations Related to Online Privacy Panic Situations. In: Eleventh Symposium On Usable Privacy and Security, SOUPS 2015:19-38.

36 Shneiderman B. The eyes have it: a task by data type taxonomy for information visualizations. In: Proceedings IEEE Symposium on Visual Languages 1996:336343. 


\section{Summary points}

What was already known:

- Existing guidelines are not sufficient to make medical dashboards usable.

- Adaptive user interfaces have been proposed to address this problem.

- Informing the design of adaptive interfaces for medical dashboards remains unexplored.

- Systems that adapt to the skills and expertise of the users should track competence automatically and unobtrusively.

What this study adds:

- We compute proxies of competence on two cohorts of users of a medication safety intervention.

- It is feasible to characterise the interactive behaviour of users in a medication safety dashboard using user interaction events.

- Primary users were more competent on population health monitoring activities, while secondary users struggled on activities involving a detailed breakdown of the safety of patients.

- Workflows that group characteristic activities of the user groups and adaptive nudges to increase user engagement. 